\title{
Hybridization of Artificial Bee Colony Algorithm and its variants with Hyperbolic Spiral based Local Search
}

\author{
Shiv Kumar Agarwal, Surendra Yadav
}

\begin{abstract}
Artificial bee colony (ABC) algorithm is grounded on intelligent swarming behavior of honey bees. It is one of the efficient algorithm for optimization. The ABC algorithm is good in exploration and sometimes fails to exploit properly. Local search strategies in addition to existing steps play important role to improve exploitation. In order to improve exploitation here a local search inspired by the nature of hyperbolic spiral introduced in $A B C$. The purposed variant used with $A B C$, Best-so-far $A B C$ and Gbest $A B C$. Outcomes proved that hybrid of these algorithms with hyperbolic search gives good results with higher accuracy and reliability.
\end{abstract}

Keywords: Local Search, Optimization, Nature Inspired Algorithm, Swarm Intelligence

\section{INTRODUCTION}

Nature has inspired human beings to develop efficient and simple approaches to get rid of intricate real world problems. Various algorithms also developed to solve numerous problems that are not answerable by conventional strategies. Nature inspired algorithms (NIA) are motivated by collective intelligent behavior of natural entities. Categorization of these algorithms depends on cause of inspiration. An algorithm may be inspired by some biological phenomenon, chemical reaction, physics based activity and many more. In recent years swarm based algorithms are most popular for solving optimization problems. Swarm based algorithms simulate collective clever behavior of simple agents. Various social insects are individually very simple with very small memory and with very limited physical strength but collectively they act like an intelligent and powerful single entity with any central control. This happens due to cooperative behavior and division of labor among these

Revised Manuscript Received on December 30, 2019.

* Correspondence Author

Shiv Kumar Agarwal*, Department of Computer Science and Engineering, Career Point University, Kota, Rajasthan, India. Email: agarwalshiv83@yahoo.co.in

Surendra Yadav, Department of Computer Science and Engineering, Career Point University, Kota, Rajasthan, India. Email: syadav66@gmail.com

(C) The Authors. Published by Blue Eyes Intelligence Engineering and Sciences Publication (BEIESP). This is an open access article under the CC BY-NC-ND license (http://creativecommons.org/licenses/by-nc-nd/4.0/) simple agents. This phenomenon may be observed when these agents search for food and safe habitat.

The honey bees also depict intelligent behavior while searching for quality food sources. Dervis Karaboga [1] observed this phenomenon and developed a novel approach namely artificial bee colony algorithm in year 2005. The ABC algorithm is one of the simplest and most prevalent meta-heuristic in field of NIAs. It is proved in literature that $A B C$ is very efficient in exploration but not as good in exploitation. In order to get precise results it is expected to devise an algorithm that is capable to balance exploration of whole search space and exploitation of best feasible solutions. To improve exploitation process in ABC, here a local search considered that exploit best solutions in search space. The newly introduced local search inspired by the nature of hyperbolic spiral. Due to its nature, hyperbolic spiral based local search (HSLS) improves the performance of $\mathrm{ABC}$ and its variants. Experimental results prove that hybrid of HSLS with basic ABC, Best-so-far ABC and Gbest $A B C$ is very successful and significantly improves the performance.

Remaining paper is as follow: Section 2 discusses ABC algorithm in brief. The Section 3 elucidates some popular variant of ABC algorithm. Section 4 shows hybrid of HSLS with existing strategies. The experimental setup and performance analysis of proposed hybrid strategies discussed in section 5. Lastly, Section 6 contains conclusion.

\section{ARtificial BeE COLONy (ABC) ALgorithm}

The ABC algorithm is defined as a set of three phases corresponding to three types of bees. A swarm of solution initialized using Eq. 1 . Solutions are bounded in lower $\left(x_{L B j}\right)$ and upper bound $\left(x_{U B j}\right)$ in $i$ dimensional search space.

$x_{i j}=x_{L B j}+\operatorname{rand}(0,1) \times\left(x_{U B j}-x_{L B j}\right)$

After initialization all the solutions update themselves with the help of Eq. 2. This phase known as employed bee phase. $v_{i j}=x_{i j}+\phi\left(x_{i j}-x_{k j}\right)$

Next phase is onlooker bee phase. During this phase bee residing in bee hive observe the behavior of onlooker bee and evaluate their performance i.e. measure the quality of solution using fitness function (refer Eq. 3).

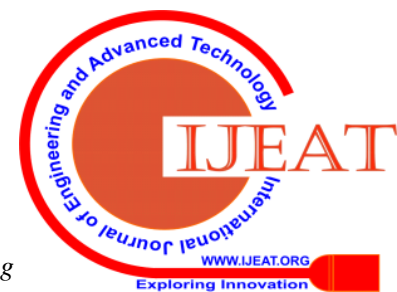


$\operatorname{Pr} o b_{i}=\frac{\text { fitness }_{i}}{\sum_{i=1}^{\text {colonySizze/2 }_{\text {fitness }}}}$

Where fitness of an individual computed by Eq. 4 .

fitness $_{i}=\left\{\begin{array}{lr}1 /\left(1+f\left(x_{i}\right)\right) & \text { if } f\left(x_{i}\right) \geq 0, \\ 1+\operatorname{abs}\left(f\left(x_{i}\right)\right) & \text { else }\end{array}\right.$

A bee become scout bee if it is not able to improve its position after certain iterations, it should be randomly is re-initialized using Eq. (1). Detailed study of ABC algorithm carried out by Bansal et al. [2], Kumar et al. [3] and Agarwal et al. [4].

\section{PopUlar VARIANT OF ABC ALgORITHM}

The ABC algorithm is one of the best performing swarm based algorithm but due to its drawbacks it is modified number of times. Mainly performance of ABC depends on position update process, which takes place using Eq. 2. A generalized form of Eq. 2 is shown below [2]:

$v_{i j}=A \times x_{i j}+B \times\left(x_{i j}-x_{k j}\right)$

Here A and B are weights assigned to target solution and difference vector of current to random solution. These weights vary from variant to variant.

The ABC algorithm modified by Zhu et al. [5] with use of global best solution while computing difference vector and named as Gbest guided ABC (GABC) as the best solution guide all the solutions in the direction of optimal solution.

$v_{i j}=x_{i j}+\phi\left(x_{i j}-x_{k j}\right)+\psi\left(x_{G b e s t j}-x_{i j}\right)$

The best-so-far approach in ABC (BSFABC) [6] introduced three modifications in basic $\mathrm{ABC}$ : selection of best solution found so far (refer Eq. 7), adjustable radius for search space (refer Eq. 8) adaptable with iteration counter and comparison of solutions based on objective values (refer Eq. 9).

$v_{i j}=x_{i j}+\phi_{i j}\left[w_{\max }-\left(\frac{\text { iteration }}{M C N}\right)\left(w_{\max }-w_{\min }\right)\right] x_{i j}$

(8)

Fitness $(f(x))= \begin{cases}\frac{1}{1+f(x)} & \text { if } f(x)>0 \\ 1+|f(x)| & \text { if } f(x)<0\end{cases}$

These two variant of ABC algorithm are very popular due to their applicability at wide range of real world applications. Gbest ABC modified by Sharma et al. [7] with the concept of local and global best solution and get good results. Local search in ABC improved its performance like Sharma et al. [8] introduced logarithmic spiral based local search and improved Gbest ABC [9]. Bhambu et al. [10] also modified this variant and gives better results. Some position update strategies also improved its performance like Arrhenius ABC [11], Weight driven approach [12] and fitness based approach [13]. Archimedean spiral based local search in ABC also $v_{i d}=x_{i j}+\phi f_{b}\left(x_{i j}-x_{b j}\right)$

proposed by Sharma et al. [14] that is inspired by the behavior of this natural spiral and it gives good results for the considered problems. Here these two variants are selected for hybridization with hyperbolic search that is discussed in subsequent section.

\section{HYPERBOLIC SEARCH IN POPULAR VARIANT OF ABC ALGORITHM}

Hyperbolic search inspired by the nature of hyperbolic spiral that belongs to the family of Cote's spiral [15].Nature shows various types of spiral pattern that are encoded in humans, plants, galaxies, animals and many nonliving things. Fig. 1 depicts few examples of natural spirals. The new local search approach is encouraged by the nature of hyperbolic spiral that attempts to grasp the pole from infinity and wind with growing speed while touching the pole. The local search based on progress of hyperbolic spiral improves the exploitation capability of ABC and its variants.

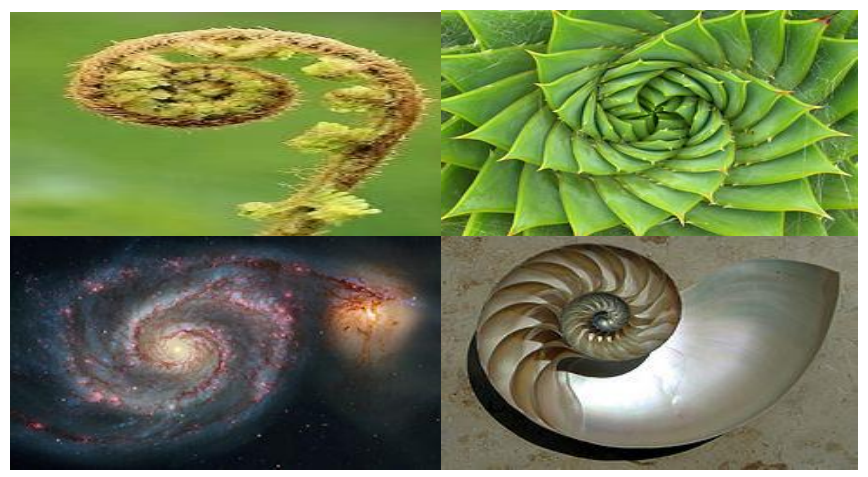

Figure 1: Spiral depicted by nature [16]

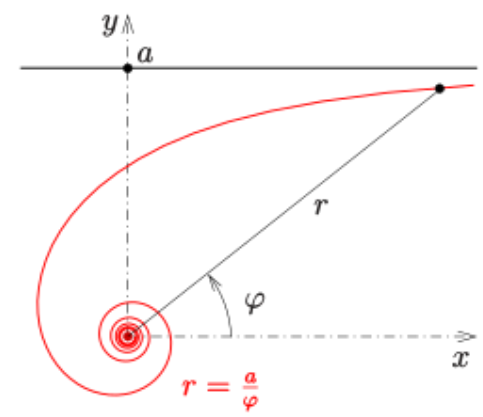

Figure 2: Hyperbolic spiral for $\theta>0$ [17]

The new local search phase based on hyperbolic search added after getting result from ordinary algorithm. The new approach updates position using Eq. 10.

$x_{i j}^{\prime}=x_{\text {best }}+s s$

$S S=\frac{\operatorname{sign} \times 2 \times U(0,1) \times\left(1-\frac{\text { Iter }}{T_{\text {_iter }}}\right)}{\sin (\text { Iter })}$

Where $T$ _iter and Iter are total iteration and iteration counter respectively. Sign decides the direction of movement.

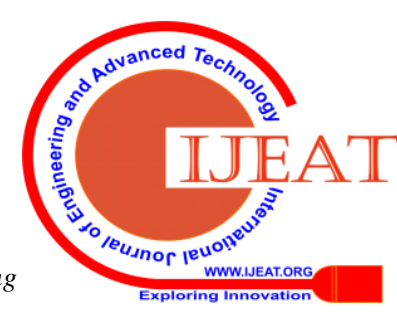


Here for the purpose of this study Gbest guided ABC and Best so far ABC are selected and hybridized with hyperbolic local search.

\section{EXPERIMENTAL SETTING AND RESULT ANALYSIS}

The performance of new hybridized algorithm is verified over a set of ten CEC standard problems. The considered problem has different characteristics. The considered functions are: Griewank $\left(\mathrm{f}_{1}\right)$, Ackley $\left(\mathrm{f}_{2}\right)$, Alpine $\left(\mathrm{f}_{3}\right)$, Michalewicz $\left(\mathrm{f}_{4}\right)$, Cosine Mixture $\left(\mathrm{f}_{5}\right)$, Exponential $\left(\mathrm{f}_{6}\right)$, Salomon Problem $\left(\mathrm{f}_{7}\right)$, Inverted cosine wave function $\left(\mathrm{f}_{8}\right)$, Neumaier 3 Problem $\left(f_{9}\right)$ and Colville function $\left(f_{10}\right)$. To demonstrate that new algorithm is best option in comparison to other existing variants, it is equated on the basis of success rate (SR) in 100 evaluations and average number of function evaluations (AFE). All the considered algorithms adopted following parameter settings.

\section{A. Experimental Setting}

All the six algorithms HSABC, GABC with HSLS, BSFABC with HSLS, ABC [1], BSFABC [5], Gbest ABC [4] are examined with the below mentioned experimental settings:

- Population size $=50$

- Criteria for termination $=200000$ iteration

- Total run $=100$

- Total iteration for local search $=10$

\section{B. Results Analysis}

Experimental results of HSABC, GABC with HSLS, BSFABC with HSLS, ABC, BSFABC, Gbest ABC recorded in terms of AFE and SR. Table 1 and Table 2 shows the numerical results for experiments and it is clearly visible from these tables that hyperbolic spiral based local search improve the performance of $\mathrm{ABC}$ and its variants.

\section{Statistical Analysis}

In order to analyze performance of proposed approach some more statistical tests are conducted. Here boxplot [18] graph shown in Figure 3 depicts that hybrid of HSLS takes very less time and converges faster than basic ABC.

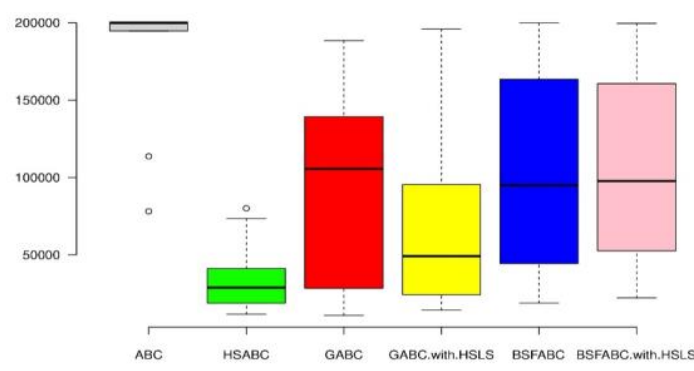

Figure 3: Boxplot Graph

Table 3 shows another comparison based on acceleration rate (AR). AR computed by using function evaluations with the help of Eq. (12).

$$
A R=\frac{\text { Function_Evaluation }_{A}}{\text { Function_Evaluation }_{\text {HSABC }}}
$$

Where A includes considered algorithms ABC, BSFABC, Gbest ABC and hybrid with HSLS.

The HSABC outperform ABC and BSFABC, while GABC is faster in case of two functions in terms of acceleration rate.

Table 1: Comparison based on AFE

\begin{tabular}{|c|c|c|c|c|c|c|}
\hline Test Function & ABC & HSABC & GABC & $\begin{array}{c}\text { GABC with } \\
\text { HSLS }\end{array}$ & BSFABC & $\begin{array}{c}\text { BSFABC with } \\
\text { HSLS }\end{array}$ \\
\hline $\mathrm{f}_{1}$ & 113690.46 & $\mathbf{3 0 4 1 4 . 1 1}$ & 139419.5 & 42355.4 & 63511.08 & 69968.99 \\
\hline $\mathrm{f}_{2}$ & 78153.5 & $\mathbf{2 7 6 1 8}$ & 28428.18 & 35883.2 & 72675 & 83416.4 \\
\hline $\mathrm{f}_{3}$ & 199725 & $\mathbf{7 3 4 9 1}$ & 107385.9 & $\mathbf{7 0 2 2 9 . 7 6}$ & 145072.5 & 172415.72 \\
\hline $\mathrm{f}_{4}$ & 200032.35 & 30101.3 & 47640.26 & $\mathbf{2 4 2 9 6 . 7 5}$ & 46802.54 & 52637.51 \\
\hline $\mathrm{f}_{5}$ & 200039.93 & 25597.5 & $\mathbf{1 3 9 5 0}$ & 18387.2 & 31618.5 & 38268.2 \\
\hline $\mathrm{f}_{6}$ & 200027.3 & 11596.2 & $\mathbf{1 0 8 8 2 . 5}$ & 14312 & 18737 & 22212.8 \\
\hline $\mathrm{f}_{7}$ & 194666.53 & $\mathbf{1 6 8 3 1 . 9}$ & 152381.8 & 95493.6 & 184747.81 & 112100.28 \\
\hline $\mathrm{f}_{8}$ & 200026.32 & $\mathbf{4 1 1 7 9 . 8 7}$ & 103805.4 & 55943.6 & 128887.3 & 131998.22 \\
\hline $\mathrm{f}_{9}$ & 200023.26 & $\mathbf{8 0 0 8 7 . 4 6}$ & 188444.6 & 195942.71 & 200025.54 & 19615.74 \\
\hline $\mathrm{f}_{10}$ & 200019.35 & $\mathbf{1 8 7 1 7 . 7 2}$ & 128206.8 & 169722.28 & 167616.76 & 160644.77 \\
\hline
\end{tabular}


Table 2: Comparison based on SR

\begin{tabular}{|c|c|c|c|c|c|c|}
\hline $\begin{array}{c}\text { Test } \\
\text { Function }\end{array}$ & ABC & HSABC & GABC & $\begin{array}{c}\text { GABC with } \\
\text { HSLS }\end{array}$ & BSFABC & $\begin{array}{c}\text { BSFABC } \\
\text { with HSLS }\end{array}$ \\
\hline$f_{1}$ & 70 & 98 & 58 & 100 & 99 & 98 \\
\hline$f_{2}$ & 100 & 100 & 100 & 100 & 100 & 100 \\
\hline$f_{3}$ & 1 & 100 & 93 & 99 & 96 & 88 \\
\hline$f_{4}$ & 0 & 100 & 100 & 100 & 100 & 100 \\
\hline$f_{5}$ & 0 & 100 & 100 & 100 & 100 & 100 \\
\hline$f_{6}$ & 0 & 100 & 100 & 100 & 100 & 100 \\
\hline$f_{7}$ & 11 & 100 & 64 & 95 & 73 & 95 \\
\hline$f_{8}$ & 0 & 100 & 88 & 100 & 84 & 78 \\
\hline$f_{9}$ & 0 & 100 & 12 & 5 & 1 & 1 \\
\hline$f_{10}$ & 0 & 100 & 61 & 35 & 37 & 38 \\
\hline
\end{tabular}

Table 3: Comparison based on AR of HSABC

\begin{tabular}{|c|c|c|c|c|c|}
\hline $\begin{array}{c}\text { Test } \\
\text { Function }\end{array}$ & ABC & GABC & BSFABC & $\begin{array}{c}\text { GABC with } \\
\text { HSLS }\end{array}$ & $\begin{array}{c}\text { BSFABC with } \\
\text { HSLS }\end{array}$ \\
\hline$f_{1}$ & 3.74 & 4.58 & 2.18 & 1.39 & 2.30 \\
\hline$f_{2}$ & 2.83 & 1.03 & 2.63 & 1.30 & 3.02 \\
\hline$f_{3}$ & 2.72 & 1.46 & 1.98 & 0.96 & 2.35 \\
\hline$f_{4}$ & 6.65 & 1.58 & 1.48 & 0.81 & 1.75 \\
\hline$f_{5}$ & 7.81 & 0.54 & 1.25 & 0.72 & 1.49 \\
\hline$f_{6}$ & 17.25 & 0.94 & 1.62 & 1.23 & 1.92 \\
\hline$f_{7}$ & 11.57 & 9.05 & 11.04 & 5.67 & 3.21 \\
\hline$f_{8}$ & 4.86 & 2.52 & 2.85 & 1.36 & 2.49 \\
\hline$f_{9}$ & 2.50 & 2.35 & 2.49 & 2.45 & 8.58 \\
\hline$f_{10}$ & 10.69 & 6.85 & 8.73 & 9.07 & \\
\hline
\end{tabular}

with four constraints [10]. Main design variables are diameter

\section{IMPLEMENTATION TO REAL WORLD ENGINEERING OPTIMIZATION PROBLEMS}

To realize the robustness of the anticipated approach the hybrid of HSLS with variant of ABC used to crack three real world engineering optimization problems. The considered problems are compression spring problem, parameter estimation for FM sound wave and welded beam design problem. Bhambu et al. [10] discussed problems in detail with objective function and constraints.

The compression spring problem is targeted to design a compression spring with minimum weight subjected to some constraints [19]. It has three simple design variables of wire and coil and number of coils. These compression springs are used in various equipments of divers range like pens to automotive systems. The parameter estimation for FM sound wave is one of the complex six dimensional problems [10]. Here main target is to produce a sound similar to objective function with some minimum value.

The welded beam design problem is related to civil engineering especially in structural engineering and an optimization problem [20]. Here main aim is to design a beam with minimum cost subject to particular buckling load, shear stress, end deflection and bending stress [10].

Table 4: Result comparison based on AFE

\begin{tabular}{|c|c|c|c|}
\hline Algorithm & $\begin{array}{c}\text { Compression spring } \\
\text { problem }\end{array}$ & $\begin{array}{c}\text { Parameter estimation for } \\
\text { FM sound wave problem }\end{array}$ & $\begin{array}{c}\text { Welded beam design } \\
\text { problem }\end{array}$ \\
\hline ABC & 194781.12 & 200011.35 & 200024.81 \\
\hline GABC & $\mathbf{1 6 5 3 2 1}$ & 189764.7 & 111417.8 \\
\hline BSFABC & 198144.4 & 199476.7 & 58199.63 \\
\hline GABC with HSLS & 192297.61 & 194560.97 & 139805.32 \\
\hline BSFABC with HSLS & 199335.87 & 200011.35 & 63350.96 \\
\hline HSABC & 182448.6 & $\mathbf{9 6 1 1 8 . 6 1}$ & $\mathbf{1 0 1 8 2 . 4 9}$ \\
\hline
\end{tabular}


Table 5: Result comparison based on SR

\begin{tabular}{|c|c|c|c|}
\hline Algorithm & $\begin{array}{c}\text { Compression spring } \\
\text { problem }\end{array}$ & $\begin{array}{c}\text { Parameter estimation for } \\
\text { FM sound wave }\end{array}$ & $\begin{array}{c}\text { Welded beam design } \\
\text { problem }\end{array}$ \\
\hline ABC & 6 & 0 & 0 \\
\hline GABC & 13 & 19 & 66 \\
\hline BSFABC & 1 & 1 & 99 \\
\hline GABC with HSLS & 13 & 10 & 58 \\
\hline BSFABC with HSLS & 1 & 0 & $\mathbf{1 0 0}$ \\
\hline HSABC & $\mathbf{1 8}$ & $\mathbf{7 4}$ & 95 \\
\hline
\end{tabular}

Experimental results of HSABC, GABC with HSLS, BSFABC with HSLS, ABC, BSFABC, and Gbest ABC recorded in terms of AFE and SR. Table 4 and Table 5 illustrates the investigational outcomes of the deliberated algorithms on the engineering optimization problems and it is clearly visible from these tables that hyperbolic spiral based local search improve the performance of $\mathrm{ABC}$ and its variants for considered three real world problems.

\section{CONCLUSION}

Herein, hybrid of hyperbolic spiral based local search and ABC, GABC, BSFABC are proposed and tested over benchmark problems. Results shows that new hybrid approaches are performing very well and may be considered as an alternative for complex optimization problems. The local search strategy helps in balancing exploration and exploitation process with the aim to improve convergence rate and efficiency of ABC algorithm. The proposed modification also tested for three engineering optimization problems and results are satisfactory. In future this variant may be used to solve more complex real world optimization problems.

\section{REFERENCES}

1. D. Karaboga. An idea based on honey bee swarm for numerical optimization. Techn. Rep. TR06, Erciyes Univ. Press, Erciyes, 2005.

2. Jagdish Chand Bansal, Harish Sharma, and Shimpi Singh Jadon. Artificial bee colony algorithm: a survey. International Journal of Advanced Intelligence Paradigms, 5(1-2):123-159, 2013.

3. Kumar, S., \& Kumari, R. (2018). Artificial Bee Colony, Firefly Swarm Optimization, and Bat Algorithms. Advances in Swarm Intelligence for Optimizing Problems in Computer Science, 145-182.

4. Shiv Kumar Agarwal and Surendra Yadav. A comprehensive survey on artificial bee colony algorithm as a frontier in swarm intelligence. In Ambient Communications and Computer Systems, pages 125-134, Springer, 2019.

5. G. Zhu and S. Kwong. Gbest-guided artificial bee colony algorithm for numerical function optimization. Applied Mathematics and Computation, 217(7):3166-3173, 2010.

6. A. Banharnsakun, T. Achalakul, and B. Sirinaovakul. The best-so-far selection in artificial bee colony algorithm. Applied Soft Computing, 11(2):2888-2901, 2011.

7. Sharma, H., Sharma, S., \& Kumar, S. (2016, September). Lbest Gbest artificial bee colony algorithm. In 2016 International conference on advances in computing, communications and informatics (ICACCI) (pp. 893-898). IEEE.

8. Sonal Sharma, Sandeep Kumar, and Kavita Sharma. Improved gbest artificial bee colony algorithm for the constraints optimization problems. Evolutionary Intelligence, pages 1-7, 2019.

9. Sharma, S., Kumar, S., \& Nayyar, A. (2018, August). Logarithmic Spiral Based Local Search in Artificial Bee Colony Algorithm. In International Conference on Industrial Networks and Intelligent Systems (pp. 15-27). Springer, Cham.

10. P Bhambu, S Sharma, and S Kumar. Modified gbest artificial bee colony algorithm. In Soft Computing: Theories and Applications, pages 665-677. Springer, 2018.

11. Kumar, S., Nayyar, A.,\&Kumari,R. (2019).Arrhenius Artificial Bee Colony Algorithm. In International Conference on Innovative Computing and Communications (pp. 187-195). Springer, Singapore.

12. $\mathrm{P}$ Tiwari and S Kumar. Weight driven position update artificial bee colony algorithm. In Advances in Computing, Communication, \& Automation (ICACCA) (Fall), International Conference on, pages 1-6. IEEE, 2016.

13. Kumar, A., Kumar, S., Dhayal, K., Swetank, K. (2014). Fitness based Position Update in Artificial Bee Colony Algorithm. International Journal of Engineering Research \& Technology, 3(5), 636 - 641.

14. Sharma, S., Kumar, S., \& Sharma, K. (2019). Archimedean spiral based artificial bee colony algorithm. Journal of Statistics and Management Systems, 22(7), 1301-1313.

15. EA Bowser. An Elementary Treatise on Analytic Geometry, Embracing Plane Geometry and an Introduction to Geometry of Three Dimensions. D. Van Nostrand, 1880.

16. Bourne, M. (2011). Golden Spiral. [online] Intmath.com. Available at: https://www.intmath.com/blog/mathematics/golden-spiral-6512 [Accessed 11 Nov. 2019].

17. En.wikipedia.org. (2019). Hyperbolic spiral. [online] Available at: https://en.wikipedia.org/wiki/Hyperbolic_spiral [Accessed 11 Nov. 2019].

18. DF Williamson, RA Parker, and JS Kendrick. The box plot: a simple visual method to interpret data. Annals of internal medicine, 110(11):916, 1989.

19. Onwubolu, G. C., Babu, B. V. (2013). New optimization techniques in engineering (Vol. 141)

20. Ragsdell, K. M., Phillips, D. T. (1976). Optimal design of a class of welded structures using geometric programming. Journal of Engineering for Industry, 98(3), 1021-1025.

\section{AUTHORS PROFILE}

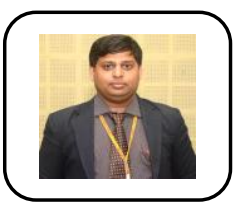

Shiv Kumar Agarwal did his B.Tech in 2006, M.Tech in 2014 and presently pursuing Ph.D from Career Point University, Kota, India. He has more than 13 years of teaching experience in Engineering Colleges. His key areas are Data base management system, Information Security and Soft Computing. His research interest includes swarm intelligence algorithms and their applications in various complex engineering design problems. His publications are in Journals and International Conferences of repute.

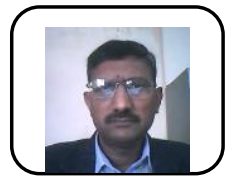

Surendra Yadav is working as a Professor in Department of Computer Science and Engineering, Career Point University, Kota, India. He obtained his Ph.D in Computer Science from Rajasthan University, Jaipur, India. He has more than 20 years of teaching experience in Engineering Colleges. His key areas are Data Mining, Information Security and Soft Computing and Big Data. His research interest includes swarm intelligence algorithms and their applications in various complex engineering design problems. His publications are in Journals and International Conferences of repute.

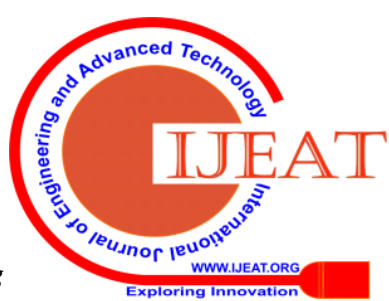

